\title{
Impairment of clathrin-mediated endocytosis via cytoskeletal change by epithelial to fibroblastoid conversion in HepG2 cells: A possible mechanism of des- $\gamma$-carboxy prothrombin production in hepatocellular carcinoma
}

\author{
KAZUMOTO MURATA and ATSUSHI SAKAMOTO \\ Center for Community Medicine, Jichi Medical University, Shimotsuke, Tochigi 329-0498, Japan
}

Received July 7, 2008; Accepted August 24, 2008

DOI: 10.3892/ijo_00000104

\begin{abstract}
Des- $\gamma$-carboxy prothrombin (DCP) has been well established as a hepatocellular carcinoma (HCC) tumor marker. However, the precise mechanism by which HCC cells produce DCP remains unknown. Importantly, DCP is not specific for HCC. For example, vitamin K-deficiency or ingestion of a vitamin $\mathrm{K}$ antagonist (warfarin) also leads to DCP production. In addition, supplementary administration of vitamin K2 analogues to HCC patients has led to reduce serum DCP levels. From these observations, we hypothesize that DCP might be produced from HCC cells with functional impairment of vitamin $\mathrm{K}$ uptake. Because, as previously reported, the down-regulation of E-cadherin or high serum DCP in HCC patients is associated with a high risk of vascular invasion, intra-hepatic metastasis and tumor recurrence, we examined if HCC cells might produce DCP by epithelial to fibroblastoid conversion (EFC) in vitro. HepG2 cells were induced EFC by tumor promoter, 12- $O$-tetracanoylphorbol13-acetate (TPA). DCP production was observed in HepG2 cells that had lost E-cadherin expression in a TPA-dosedependent manner. The DCP production was inhibited by introducing additional vitamin K2 into the treated cells. In addition, LDL uptake as a surrogate of vitamin $\mathrm{K}$ uptake was significantly impaired in TPA-treated HepG2 cells. The cells with impairment of LDL uptake produced DCP. Fat soluble vitamins are taken up into cells through clathrin-mediated endocytosis, in which the dynamic polymerization of F-actin plays a crucial role. We found that HepG2 cells with F-actin rearrangement produced DCP. In addition, latrunculin A, an actin depolymerizer, induced naïve HepG2 cells to produce $\mathrm{DCP}$, confirming that impairment of F-actin polymerization
\end{abstract}

Correspondence to: Dr Kazumoto Murata, Center for Community Medicine, Jichi Medical University, 3311-1 Yakushiji, Shimotsuke, Tochigi 329-0498, Japan

E-mail: atarum@jichi.ac.jp

Key words: des- $\gamma$-carboxy prothrombin, hepatocellular carcinoma, vitamin K, clathrin-mediated endocytosis, epithelial to fibroblastoid conversion is a key mechanism of DCP production. We showed in vitro that cytoskeletal filament change by EFC is crucial for DCP production in HepG2 cells.

\section{Introduction}

Prothrombin is a vitamin $\mathrm{K}$-dependent blood coagulation protein that is synthesized in the liver, and biological active form of prothrombin is produced when vitamin $\mathrm{K}$ functions as a cofactor in the posttranslational carboxylation of specific glutamyl residues. In the absence of vitamin $\mathrm{K}$ or when its action is antagonized by warfarin, abnormal prothrombin (des- $\gamma$-carboxy prothrombin; DCP), also known as 'protein induced by vitamin $\mathrm{K}$ absence or antagonist II' (PIVKA-II), is released into the blood (1). DCP also has been well established as a hepatocellular carcinoma (HCC) tumor marker (2), and several authors have suggested the mechanism of DCP production in HCC cells such as reduction of $\gamma$-carboxylase gene expression (3), excessive synthesis of prothrombin precursors (4) or reduction of vitamin $\mathrm{K}$ content (5). However, the precise mechanism of DCP production by $\mathrm{HCC}$ remains unclear. Importantly, DCP is not specific for HCC, and DCP antigen in patients with HCC is thought to be similar to that in patients who are treated with warfarin (1). In addition, supplementary administration of vitamin $\mathrm{K} 2$ analogues to HCC patients reduced serum DCP levels (6), and vitamin K2 inhibited DCP production in vitro as well (7).

Recent clinical evidence suggests that high serum DCP in HCC patients is associated with a high risk of vascular invasion $(8,9)$, intra-hepatic metastasis $(9)$ and tumor recurrence (10). Down-regulation of E-cadherin is involved in the early recurrence of HCC after surgical treatment (11) or metastasis (12). Thus, several lines of clinical evidence suggest that migration or invasion of tumor cells is prompted by the loss of E-cadherin, which is a central component of cell-cell adhesion junctions. In addition, the juxtamembrane region of cadherins may signal to the actin cytoskeleton, given that E-cadherin is attached to the cytoskeleton via connections to several cytoplasmic proteins such as $ß$-catenins (13). Actually, the cytoskeleton is dramatically reorganized during epithelial-mesenchymal transition (EMT) (14), and epithelial to fibroblastoid conversion (EFC) in HCC is accompanied by extensive reprogramming of the cytoskeleton (15). In addition, 
actin-based cytoskeleton reorganization affects several processes that need to reshape the plasma membrane, including different forms of endocytic internalization and the protrusion of lamellipodia and filopodia during cell migration (16-18). From these pieces of evidence, we hypothesized that cytoskeletal filaments change during EFC is crucial for DCP production by impairment of vitamin $\mathrm{K}$ uptake.

\section{Materials and methods}

Cell culture. HepG2 cells were maintained in Dulbecco's modified Eagle's medium (DMEM) containing $10 \%$ fetal calf serum (10\% DMEM). For EFC, cells were incubated with 12-O-tetracanoylphorbol-13-acetate (TPA) for $24 \mathrm{~h}$ (19). For the immunofluorescence study, HepG2 cells were cultured in chamber slides (Nagle Nunc International, Rochester, NY) with $0.1 \%$ dimethyl sulfoxide (DMSO) (control) or $50 \mathrm{nM}$ TPA for $24 \mathrm{~h}$. All chemical reagents were purchased from Sigma (St. Louis, MO).

Cell migration assay. HepG2 cells were seeded into the upper chamber of a 24-well culture insert membrane (pore size; $8 \mu \mathrm{m}$, Millipore, Billerica, MA, USA) in 10\% DMEM. The cells were incubated with $0.1 \%$ DMSO or $50 \mathrm{nM}$ TPA for $24 \mathrm{~h}$. Cells that had migrated to the underside of the insert membrane were stained with $0.3 \%$ crystal violet (Sigma). The cells on the upper side of the insert membrane were gently rubbed with a cotton swab.

Western blot analysis. Cells were resolved using SDS-PAGE and transferred to nitrocellulose membranes. The membranes were incubated with anti-mouse E-cadherin $(1: 2,500, \mathrm{BD}$ Transduction Laboratories, San Jose, CA). Antibodies against B-actin (1:5,000, Abcam, Tokyo, Japan) were used for an internal control. The proteins were detected using electrochemiluminescence techniques (Pierce Chemicals, IL).

Immunofluorescence-microscopy. After cells were fixed with $4 \%$ paraformaldehyde, primary antibodies were applied to monolayer of the cells overnight at room temperature. Antirabbit E-cadherin (1:50, IBL Co., Fujioka, Japan) and antimouse PIVKA-II (1:100, a kind gift from Eisai, Tokyo, Japan) were used as primary antibodies. After washing, cells were incubated with FITC-conjugated anti-rabbit IgG (Sigma) or AlexaFluors 568-conjugated anti-mouse IgG (Invitrogen, Carlsbad, CA). The cell nuclei were stained with mounting medium containing DAPI (Vector, Burlingame, CA). FITCconjugated phalloidin (Sigma) was applied for $40 \mathrm{~min}$ at room temperature to reveal filamentous actin. Images were obtained using the 'All-In-One Immunofluorescence Microscopy' system (Keyence Inc., Tokyo, Japan).

Enzyme-linked immunosorbent assay (ELISA). HepG2 cells were seeded at a density of $2 \times 10^{5}$ cells into 24 -well plates. The next day, the medium was replaced with medium containing various concentrations of TPA, or $50 \mathrm{nM}$ TPA with various concentration of vitamin $\mathrm{K} 2$, and the cells were cultured for another $24 \mathrm{~h}$. In other experiments, HepG2 cells were cultured with $1 \mu \mathrm{M}$ latrunculin A (Lat A) with or without $100 \mathrm{nM}$ vitamin $\mathrm{K} 2$ for $24 \mathrm{~h}$. The supernatant was subjected to ELISA. Control cells were incubated with $10 \%$ DMEM containing $0.1 \%$ DMSO. The DCP concentration was determined using a PIVKA-II ELISA kit (Eisai, Tokyo, Japan) according to the manufacturer's instructions.

Uptake of low-density lipoprotein (LDL). HepG2 cells were cultured in serum free DMEM containing $1 \%$ bovine serum albumin with $0.1 \%$ DMSO or $50 \mathrm{nM}$ of TPA for $24 \mathrm{~h}$. Cells were incubated with $1 \mu \mathrm{g} / \mathrm{ml}$ of 3,3'-dioctadecylindocarbocyanin (Dil)-LDL (Biomedical Technologies, Stoughton, MA) and $10 \mu \mathrm{g} / \mathrm{ml}$ of human LDL for $20 \mathrm{~min}$ at $37^{\circ} \mathrm{C}$, then fixed with $4 \%$ paraformaldehyde. Anti-mouse PIVKA-II was applied overnight at room temperature. Monolayer was incubated with FITC-conjugated anti-mouse IgG.

Fluorescence-activated cell-sorting (FACS) analysis. HepG2 cells were cultured in serum-free DMEM containing $1 \%$ bovine serum albumin with $0.1 \%$ DMSO (control) or $50 \mathrm{nM}$ TPA for $24 \mathrm{~h}$. After being harvested, cells were incubated with human LDL receptor antibody (Acris-antibodies, Hiddenhausen, Germany) on ice for $15 \mathrm{~min}$. After washing, FITC-conjugated anti-rabbit IgG (Sigma) was applied. After fixed with $4 \%$ paraformaldehyde, cells were subjected to FACS analysis. The stained cells were analyzed using FACSCalibur and Cell Quest software (Becton-Dickinson, San Jose, CA).

Filamentous $(F)$ actin/globular $(G)$ actin in vivo assay. The ratio of F-actin versus G-actin in cells was analyzed using a G-actin/F-actin in vivo assay kit (Cytoskeleton Inc., Denver, $\mathrm{CO}$ ) according to the manufacturer's instructions. The ratio of F-actin versus $\mathrm{G}$-actin was quantified using the quantity one software (Bio-Rad, Hercules, CA).

Statistical analysis. All data are expressed as mean \pm SD. Multiple comparisons were performed using One-way ANOVA. Intergroup comparisons were performed using paired t-tests and Bonferroni's correction for multiple comparisons. Values of $\mathrm{p}<0.05$ were considered to be statistically significant.

\section{Results}

Induction of EFC in HepG2 cells. To induce EFC, we used TPA because it is known to trigger the morphological transition from epithelial to fibroblastic appearance accompanied by scattering and migration of HepG2 cells with a reduction in E-cadherin expression (19). Actually, TPA treatment caused the HepG2 cells to become scattered and to adopt a fibroblastlike appearance, whereas control cells showed round-shaped and clustered appearance (Fig. 1A). TPA treatment also induced cells to migrate to the underside of the insert membrane within $24 \mathrm{~h}$, whereas few control cells migrated in this way (Fig. 1B). These observations were quantitatively confirmed by Western blotting. In TPA-treated HepG2 cells, the expression level of E-cadherin was significantly downregulated relative to control cells (Fig. 1C).

DCP production by EFC-induced HepG2 cells. Continuous liner staining of E-cadherin along cell boundaries was observed 
Control

A

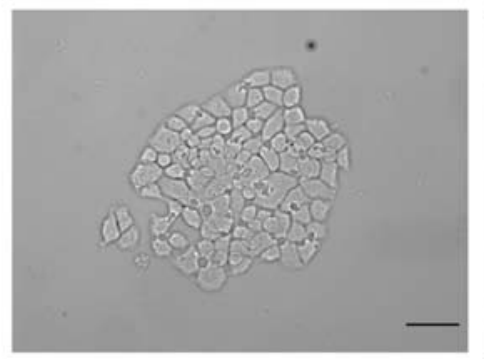

B

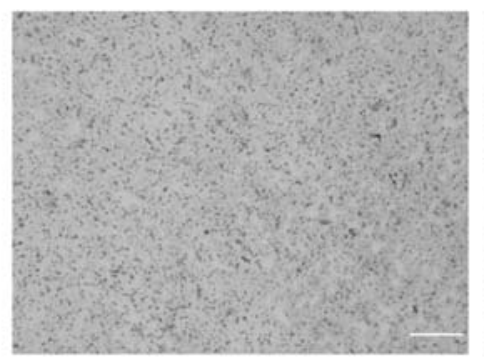

TPA
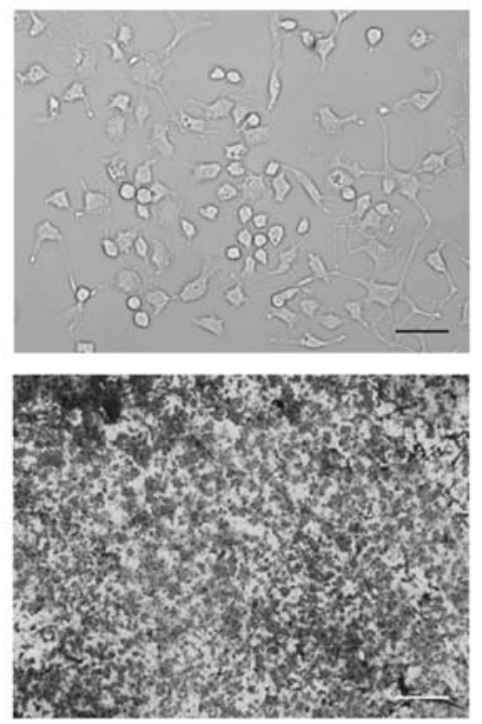

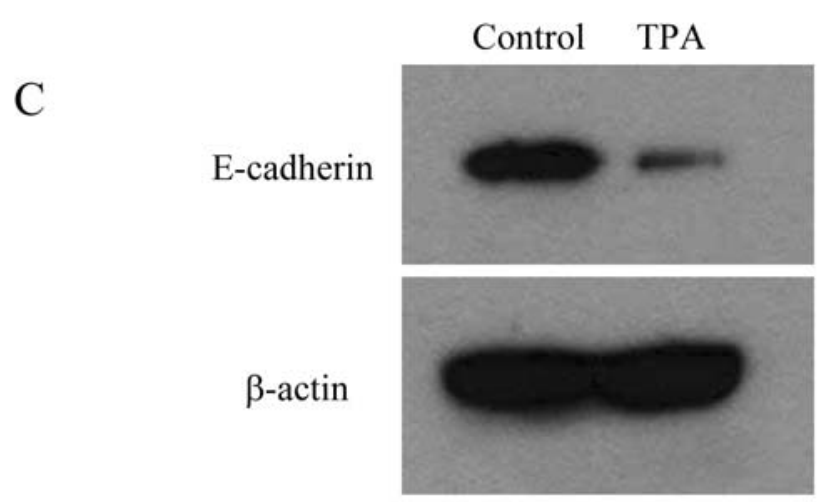

Figure 1. Cell scattering and migration by TPA treatment. (A) TPA treatment induced HepG2 cells to undergo morphological change from clustered round cells (control) to scattered fibroblast-like cells. Bars represent $100 \mu \mathrm{m}$. (B) TPA-treated HepG2 cells migrated to the under-side of the culture insert membrane, whereas few control cells migrated in this way. Bars represent $500 \mu \mathrm{m}$. (C) Representative Western blot analysis with E-cadherin.

in control HepG2 cells, whereas E-cadherin immunostaining was reduced and sparsely retained in the cytoplasm of TPAtreated HepG2 cells (Fig. 2A). Interestingly, DCP production was clearly observed in cells that lost linear E-cadherin expression (Fig. 2B). Naive HepG2 cells had different phenotypes with respect to cell morphology and DCP production. Some naïve HepG2 cells that showed fibroblast-like morphology with scattering tendency produced low level of DCP in immunofluorescence study (data not shown) although they were in the minority, whereas round-shaped and clustered HepG2 cells (typical naïve HepG2 cells) did not produce DCP.

Dose-dependent production of DCP by TPA treatment and inhibition of DCP production by additional vitamin $K 2$. The results of ELISA demonstrated that TPA induced HepG2 cells to produce DCP in a dose-dependent manner (Fig. 3A). These effects of TPA were not due to a change of cell numbers (Fig. 3B). Considered together with the results of Fig. 2, HepG2 cells produced DCP during EFC. DCP production in the TPA-treated HepG2 cells was inhibited by vitamin K2 in a dose-dependent manner (Fig. 3C) just as serum DCP is reduced by administration of vitamin $\mathrm{K} 2$ analogues in patients with HCC (6), which reminded us that these cells have functional impairment of vitamin $\mathrm{K}$ uptake, not complete destruction of DCP production pathway.

Impairment of LDL uptake in EFC-induced HepG2 cells. TPA treatment impaired Dil-LDL uptake into HepG2 cells (Fig. 4A) whereas control cells clearly internalized and accumulated Dil-LDL. In addition, the cells that had impaired Dil-LDL uptake produced DCP (Fig. 4B). Since FACS analysis demonstrated that TPA up-regulated LDL receptors on HepG2 cells (Fig. 4C) as previously reported (20), impairment of LDL-uptake by TPA may not be due to a reduction of the number of LDL receptors. These observations were consistent with previous report that vitamin $\mathrm{K}$ content was significantly reduced in $\mathrm{HCC}$ (5).

Disruption of filamentous actin in EFC-induced HepG2 cells, which resulted in DCP production. Immunofluorescence study using FITC-conjugated phalloidin revealed a subcortical typical 
Control

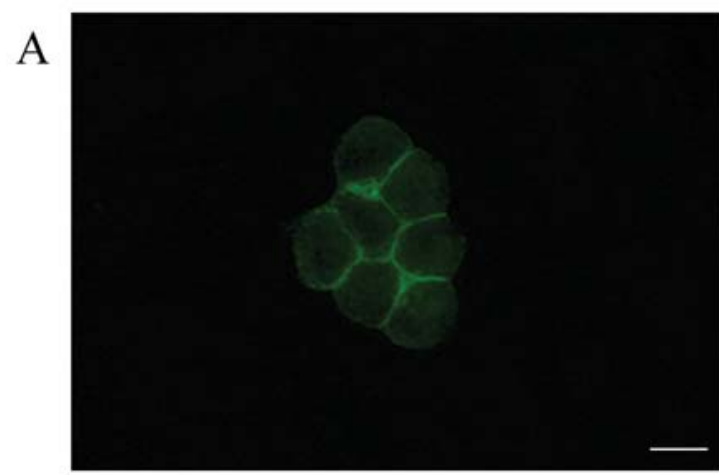

$\mathrm{B}$

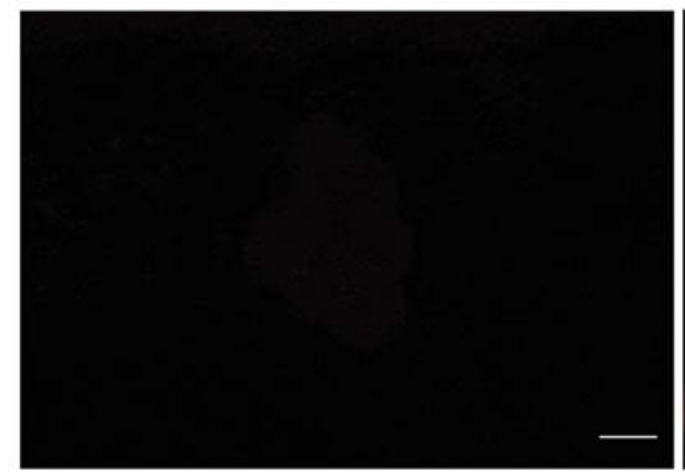

TPA
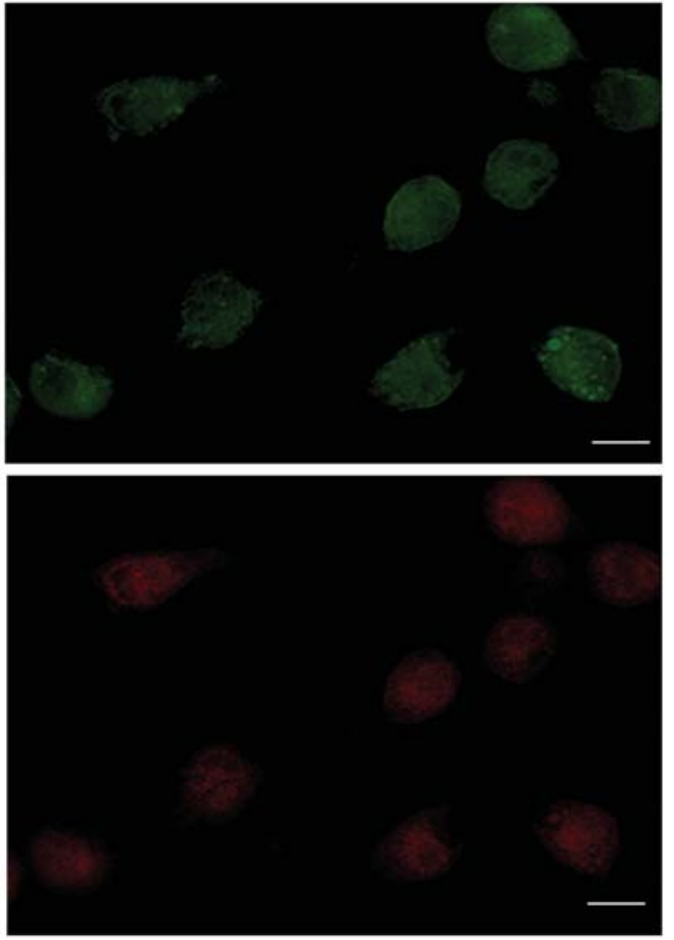

Figure 2. TPA-treated HepG2 cells produced DCP. (A and B) Double staining of E-cadherin and DCP. Linear E-cadherin (green) along cell boundaries was present in control cells, but not in TPA-treated HepG2 cells. DCP (red) was observed in TPA-treated HepG2 cells in which linear E-cadherin expression was absent. The white bars represent $20 \mu \mathrm{m}$.

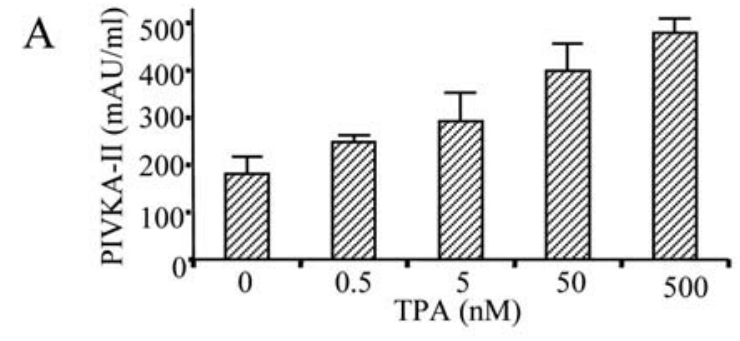

B
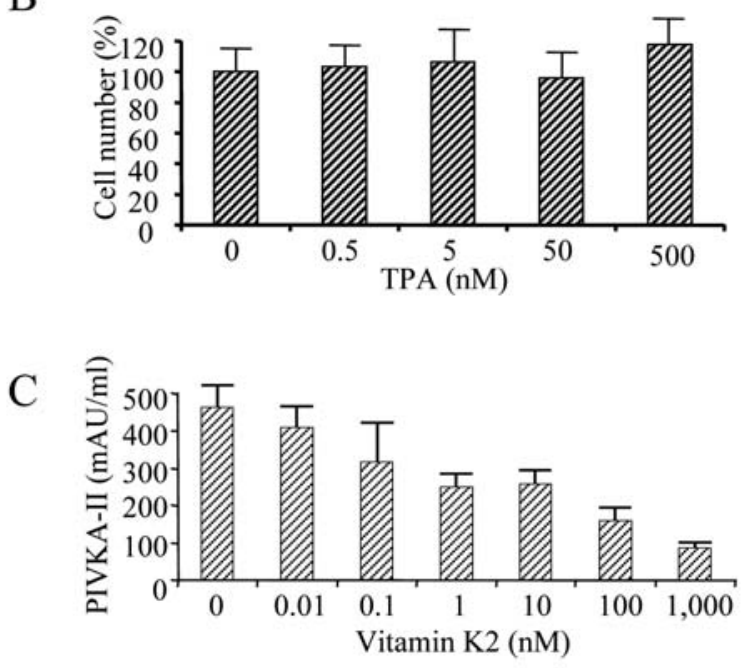

Figure 3. Effect of TPA on DCP production. (A) TPA induced HepG2 cells to produce DCP in a dose-dependent manner $(\mathrm{p}=0.00017$, One-way ANOVA). (B) The number of HepG2 cells was not changed by TPA treatment ( $\mathrm{p}=0.32$, One-way ANOVA). (C) The DCP production induced by TPA (50 nM) was inhibited by additional vitamin $\mathrm{K} 2$ in a dose-dependent manner $(\mathrm{p}=0.00004$, One-way ANOVA). network of filaments in control HepG2 cells (Fig. 5A and B). In contrast, in TPA-treated HepG2 cells, these filaments were completely absent, and dotted phalloidin-positive fibers were observed, suggesting that F-actin was disrupted by TPA treatment. To confirm this, we determined the ratio of F-actin versus free G-actin and found that the amount of F-actin was significantly reduced in TPA-treated HepG2 cells relative to control HepG2 cells (Fig. 5D). In addition, DCP production was clearly detected in TPA-treated HepG2 cells in which linear F-actin structures were absent, whereas it was not observed in control cells that showed a fine network of F-actin (Fig. 5C). To confirm the importance of F-actin for DCP production, naïve $\mathrm{HepG} 2$ cells were treated with Lat $\mathrm{A}$, which is known to disrupt F-actin (Fig. 5E). Lat A significantly induced naïve HepG2 cells to produce DCP (Fig. 5F). These results, together with Fig. 4, clearly demonstrate that disruption of F-actin is a key mechanism of DCP production by impairment of vitamin K uptake. Interestingly, Lat A-induced DCP production was inhibited by additional vitamin K2 (Fig. 5F) as seen in TPA-treated HepG2 cells (Fig. 3C).

\section{Discussion}

We demonstrated that cytoskeletal filament change during EFC is crucial for DCP production by impairment of clathrin-mediated endocytosis in HepG2 cells.

From the clinical evidence that serum DCP is detected in non-HCC patients when warfarin is administered (2), and serum DCP is reduced by administration of vitamin K2 analogues in patients with HCC (6), we initially hypothesized that DCP is produced when vitamin K uptake is impaired in 

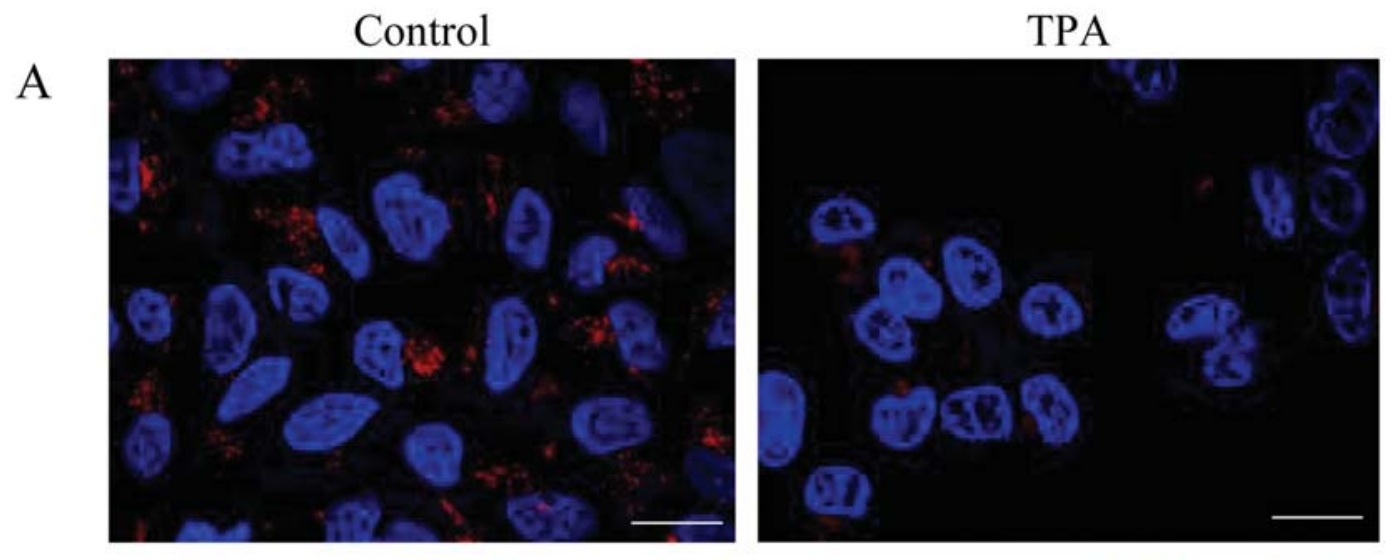

B
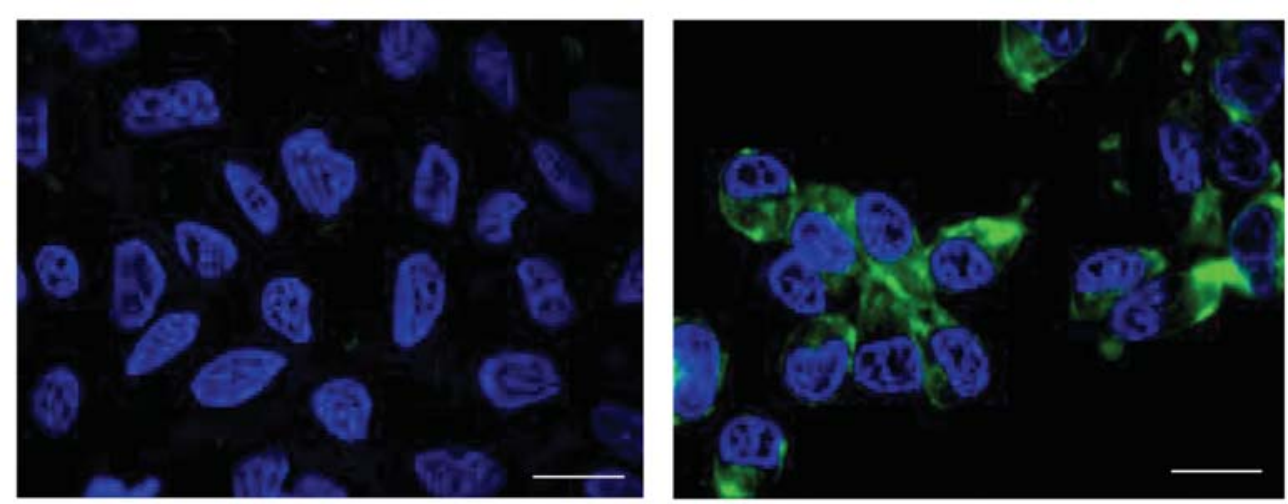

$\mathrm{C}$

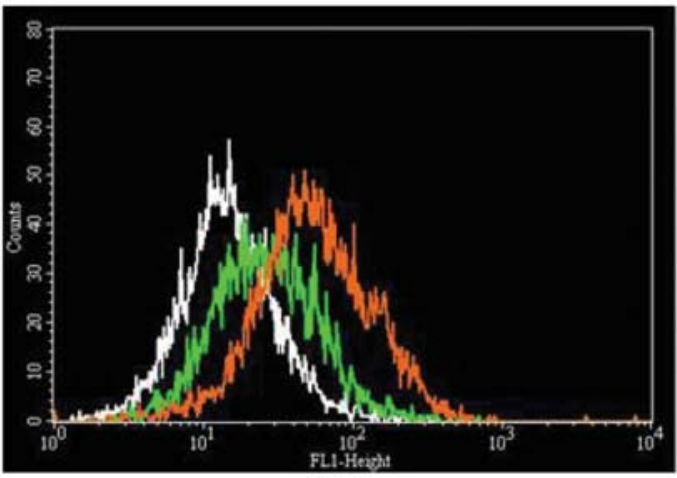

Figure 4. TPA impaired labeled LDL (Dil-LDL) uptake. (A) Dil-LDLs (red) were taken up and accumulated in control cells, whereas uptake was significantly impaired in TPA-treated HepG2 cells. (B) TPAtreated HepG2 cells with impairment of Dil-LDL uptake produced DCP (green), whereas control cells with well-uptake of Dil-LDL did not produce DCP. DAPI (blue). The white bars represent $20 \mu \mathrm{m}$. (C) FACS demonstrated that the intensity curve was shifted to the right in TPA-treated HepG2 cells (orange) relative to control HepG2 cells (green), suggesting that TPA treatment up-regulated LDL receptors. The white curve represents control cells treated with FITC-anti-rabbit $\mathrm{IgG}$, but without anti-LDL receptor.
HCC cells. However, it is difficult to examine vitamin $\mathrm{K}$ uptake itself because labeled vitamin $\mathrm{K}$ is not commercially available. Dietary vitamin K, a fat-soluble essential vitamin, is physiologically delivered to the liver in the form of chylomicron remnants (21). Furthermore, LDL receptor is essential for the normal, fast clearance of chylomicron remnants (22), and chylomicron remnants are partially taken up via LDL receptor-mediated endocytosis (23). Therefore, we used labeled LDL (Dil-LDL) and LDL uptake was actually impaired in TPA-treated HepG2 cells. In addition, LDL (vitamin $\mathrm{K}$ ) is taken up into cells through clathrin-mediated endocytic internalization (23-25). During this process, the dynamic polymerization of endogenous F-actin plays a crucial role in both mammalian and yeast cells. Therefore, we examined the relation between F-actin change and DCP production. DCP production was observed in TPA-treated HepG2 cells that disrupted F-actin. Furthermore, these observations were confirmed by the results that Lat A-treated HepG2 cells produced DCP.
The reduction of DCP production in TPA-treated HepG2 cells by additional vitamin $\mathrm{K} 2$ is supposed to be a supplementary effect of vitamin $\mathrm{K} 2$ rather than a cytotoxic effect of vitamin $\mathrm{K} 2$ because the dose of vitamin $\mathrm{K} 2$ is much lower to reduce DCP levels $(<100 \mathrm{nM})$ than that needed to inhibit HCC cell growth (50-100 $\mu \mathrm{m})(7)$. In addition, supplementary effect of vitamin $\mathrm{K} 2$ on the reduction of DCP production in both TPA- and Lat A-treated HepG2 cells suggest that the effect of both Lat A and TPA on F-actin change is partial and functional, not complete, with respect to the clathrinmediated endocytosis of LDL (vitamin K). Our result that some Dil-LDLs were observed even in TPA-treated HepG2 cells (Fig. 4A) supports this. Therefore, it is interesting to speculate that supplementary vitamin $\mathrm{K} 2$ recover total vitamin K2 uptake into Lat A-, TPA-treated HepG2 cells, resulting in reduction of DCP production. F-actin is critical for endocytosis in yeasts (26). In mammalian cells, however, F-actin has a facilitatory rather than an essential role in endocytosis (27), and inhibition of endocytosis in mammalian 

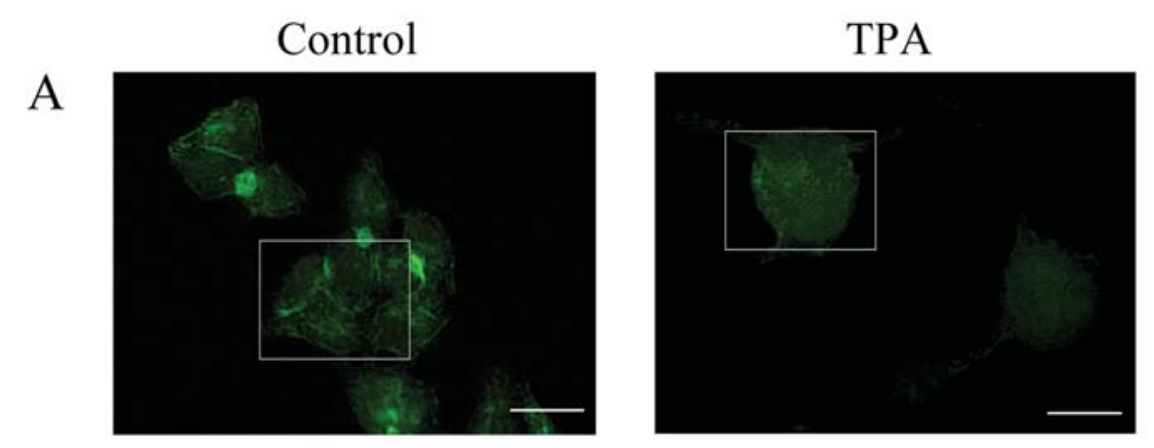

B
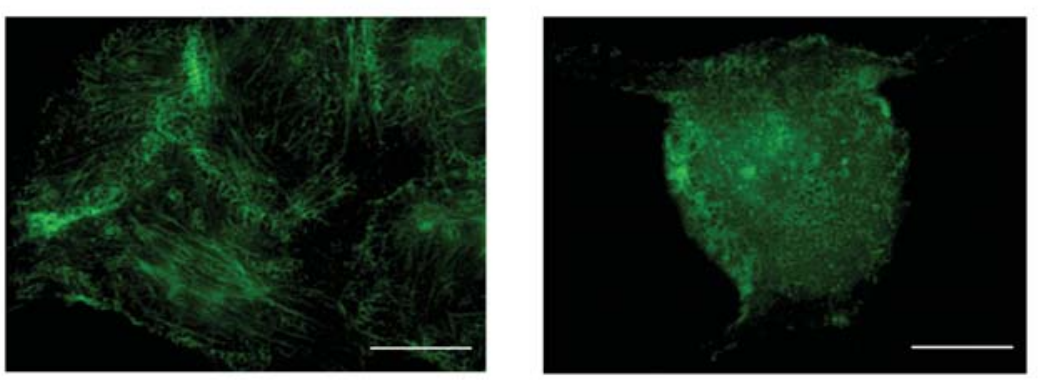

C
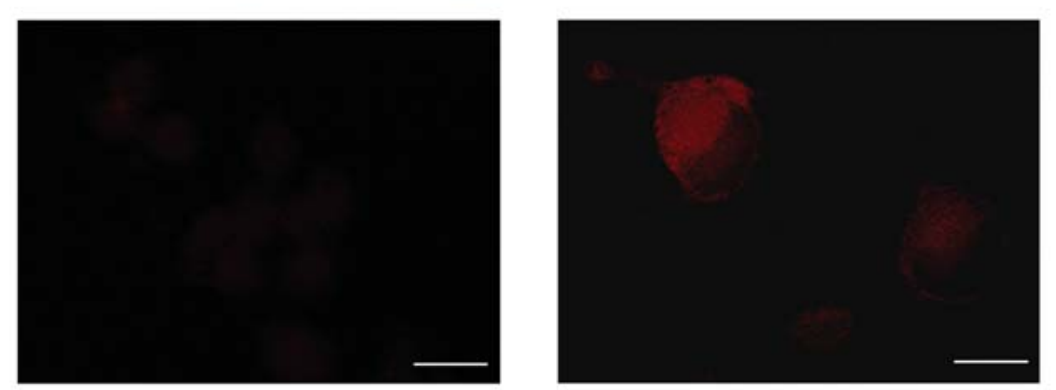

$\mathrm{D}$
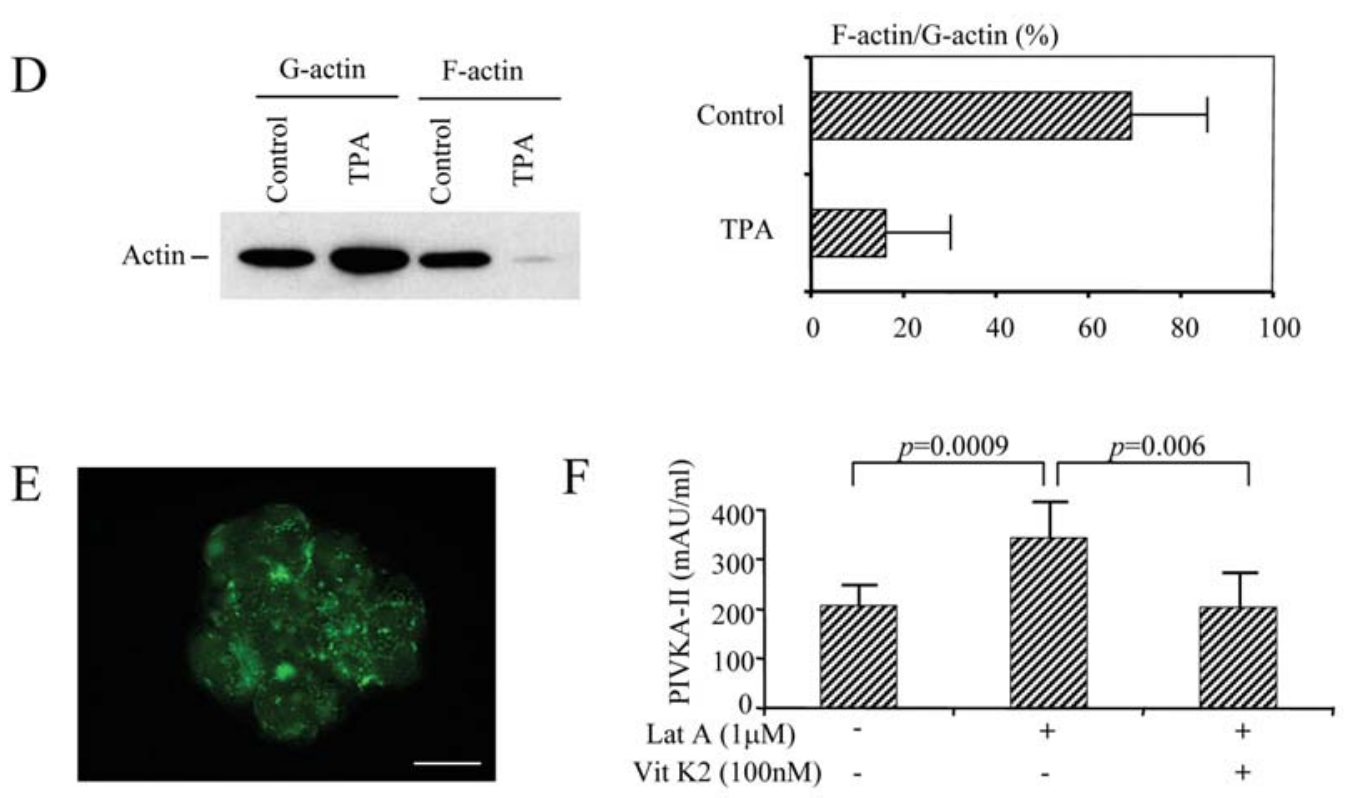

Figure 5. Disruption of F-actin and DCP production. (A) FITC-conjugated phalloidin staining showed that F-actin was disrupted in TPA-treated cells, whereas fine linear F-actin was observed in control cells. The white bars represent $20 \mu \mathrm{m}$. (B) Magnified image of the inset in A. The white bars represent $10 \mu \mathrm{m}$. (C) Double staining of phalloidin and DCP showed that TPA-treated HepG2 cells without any fine F-actin network produced DCP (red), whereas control cells with fine F-actin network did not produce DCP. The white bars represent $20 \mu \mathrm{m}$. (D) F-actin versus G-actin ratios. The amount of F-actin was significantly reduced in TPA-treated HepG2 cells relative to control cells. Bars represent means \pm SD for two independent experiments. (E) F-actin was disrupted and adopted a dotted appearance in cells treated with Lat A $(1 \mu \mathrm{M})$. The white bar represents $20 \mu \mathrm{m}$. (F) Lat $\mathrm{A}(1 \mu \mathrm{M})$ induced naïve HepG2 cells to produce DCP $(\mathrm{p}=0.0009$; paired t-test), which was inhibited by additional $100 \mathrm{nM}$ vitamin $\mathrm{K} 2$ ( $\mathrm{p}=0.006$; paired $\mathrm{t}$-test).

cells by Lat A tends to reduce the rate of internalization rather than blocking it completely (28). These pieces of evidence also support our observations.
Cell growth inhibition induced by TPA is associated with one of the mitogen-activated protein kinase, extracellular signal-related kinase (ERK), and sustained ERK activation 
is associated with cell cycle arrest via activation of cyclindependent kinase inhibitors (29). TPA treatment reduced the number of HepG2 cells in the previous report (19) whereas, in our study, it was not changed even with high dose of TPA. This discrepancy may come from cell condition. We first selected round-shaped HepG2 cells by limiting dilutions and used within 5 time division before cells would show cell scattering or fibroblastoid phenotype. Therefore, it is possible that their naïve HepG2 cells were undergoing EFC and TPA might reduce the cell number.

In conclusion, our data clarified the mechanism by which DCP is produced from HepG2 cells in vitro, showing that rearrangement of the cytoskeleton, particularly F-actin, during EFC results in functional impairment of clathrin-mediated endocytosis of vitamin K, and in turn produces DCP. However, the present study involved in vitro experiments, in which cells were chemically induced to undergo EFC, rather than natural transition. Therefore, further examinations are required to clarify if cytoskeletal rearrangement naturally occurs in vivo and these cells produce DCP.

\section{Acknowledgements}

This work was partially supported by a Grant-in-Aid for Scientific Research from the Japan Society. We thank M. Kikuchi for technical assistance with antibody selection and tissue staining. We also thank T. Gotoh, T. Oyamada and other members of Center for Community Medicine, Jichi Medical University, for technical assistance.

\section{References}

1. Blanchard RA, Furie BC, Jorgensen M, Kruger SF and Furie B: Acquired vitamin K-dependent carboxylation deficiency in liver disease. N Engl J Med 305: 242-248, 1981.

2. Liebman H, Furie BC, Tong MJ, et al: Des- $\gamma$-carboxy (abnormal) prothrombin as a serum marker of primary hepato-cellular carcinoma. N Engl J Med 310: 1427-1431, 1984.

3. Shah DJ, Engelke JA and Suttie JW: Abnormal prothrombin in the plasma of rats carrying hepatic tumors. Blood 69: 850-854 1987.

4. Ono M, Ohta H, Ohhira M, Sekiya C and Namiki M: Measurement of immunoreactive prothrombin, des-gamma-carboxy prothrombin, and vitamin $\mathrm{K}$ in human liver tissues: overproduction of immunoreactive prothrombin in hepatocellular carcinoma. Am J Gastroenterol 85: 1149-1154, 1990.

5. Huisse MG, Leclercq M, Belghiti J, et al: Mechanism of the abnormal vitamin $\mathrm{K}$-dependent gamma-carboxylation process in human hepatocellular carcinomas. Cancer 74: 1533-1541, 1994.

6. Mizuta T, Ozaki I, Eguchi Y, et al: The effect of menatetrenone a vitamin $\mathrm{K} 2$ analog, on disease recurrence and survival in patients with hepatocellular carcinoma after curative treatment. Cancer 106: 867-872, 2006.

7. Wang Z, Wang M, Finn F and Carr BI: The growth inhibitory effects of vitamin $\mathrm{K}$ and their actions on gene expression. Hepatology 22: 876-882, 1995.

8. Koike Y, Shiratori Y, Sato S, et al: Des- $\gamma$-carboxy prothrombin as a useful predisposing factor for the development of portal venous invasion in patients with hepatocellular carcinoma. Cancer 91: 561-569, 2001

9. Suehiro T, Matsumata T, Itasaka H, Taketomi A, Yamamoto K and Sugimachi K: Des-gamma-carboxy prothrombin and proliferative activity of hepatocellular carcinoma. Surgery 117: 682-691, 1995
10. Shimada M, Yonemura Y, Ijichi H, et al: Living donor liver transplantation for hepatocellular carcinoma: a special reference to a preoperative Des-gamma-carboxy prothrombin value. Transplant Proc 37: 1177-1179, 2005.

11. Inayoshi J, Ichida T, Sugitani S, et al: Gross appearance of hepatocellular carcinoma reflects E-cadherin expression and risk of early recurrence after surgical treatment. J Gastroenterol Hepatol 18: 673-677, 2003.

12. Lee TK, Poon RTP, Yuen AP, et al: Twist overexpression correlates with hepatocellualr carcinoma metastasis through induction of epithelial-mesenchymal transition. Clin Cancer Res 12: $5369-5376,2006$

13. Noren NK, Liu BP, Burridge K and Kreft B: p120 catenin regulates the actin cytoskeleton via Rho GTPases. J Cell Biol 150: 567-579, 2000

14. Savagner P: Leaving the neighborhood: molecular mechanisms involved during epithelial-mesenchymal transition. Bioessays 23: 912-923, 2001.

15. Gotzmann J, Huber H, Thallinger C, et al: Hepatocytes convert to a fibroblastoid phenotype through the cooperation of TGF- $\beta 1$ and Ha-Ras: steps towards invasiveness. J Cell Sci 115: 1189-1202, 2002.

16. Thiery JP and Sleeman JP: Complex networks orchestrate epithelial-mesenchymal transitions. Nat Rev Mol Cell Biol 7 : 131-142, 2006.

17. Kaksonen M, Toret CP and Durbin DG: Harnessing actin dynamics for clathrin-mediated endocytosis. Nat Rev Mol Cell Biol 7: 404-414, 2006.

18. Engqvist-Goldstein AE and Drubin DG: Actin assembly and endocytosis: from yeast to mammalians. Annu Rev Cell Dev Biol 19: 287-332, 2003

19. Wu WS, Tsai RK, Chang CH, Wang S, Wu JR and Chang YX: Reactive oxygen species mediated sustained activation of protein kinase $\mathrm{C} \alpha$ and extracellular signal-regulated kinase for migration of human hepatoma cell HepG2. Mol Cancer Res 4: 747-758, 2006.

20. Wilson GM, Roberts EA and Deeley RG: Modulation of LDL receptor mRNA stability by phorbol esters in human liver cell culture model. J Lipid Res 38: 437-446, 1997.

21. Newman P and Shearer MJ: Vitamin K metabolism. In Subcellular Biochemistry. Quin and Kagan (eds.) Plenum Press New York, pp455-488, 1988

22. Mortimer BC, Beveridge DJ, Martins IJ and Redgrave TG: Intracellular localization and metabolism of chylomicron remnants in the livers of LDL receptor-deficient mice and apoE-deficient mice. Evidence for slow metabolism via an alternative apoEdependent pathway. J Biol Chem 270: 28767-28776, 1995

23. Goldstein JL, Anderson RGW and Brown MS: Coated pits, coated vesicles, and receptor-mediated endocytosis. Nature 279: 679-685, 1979 .

24. Merrifield CJ, Feldman ME, Wan L and Almers W: Imaging actin and dynamin recruitment during invagination of single clathrin-coated pit. Nat Cell Biol 4: 691-698, 2002.

25. Yarar D, Waterman-Storer CM and Schmid SL: A dynamic actin cytoskeleton functions at multiple stages of clathrinmediated endocytosis. Mol Biol Cell 16: 964-975, 2005.

26. Ayscough KR, Stryker J, Pokala N, Sanders M, Crews P and Drubin DG: High rates of actin filament turnover in budding yeast and roles for actin in establishment and maintenance of cell polarity revealed using the actin inhibitor latrunculin-A. J Cell Sci 137: 399-416, 1997.

27. Fujimoto LM, Roth R, Heuser JE and Schmid SL: Actin assembly plays a variable, but not obligatory role in receptormediated endocytosis in mammalian cells. Traffic 1: 161-171, 2000.

28. Smythe E and Ayscough KR: Actin regulation in endocytosis. J Cell Sci 119: 4589-4598, 2006.

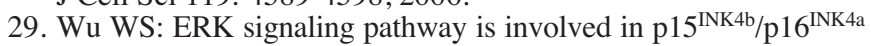
expression and HepG2 growth inhibition triggered by TPA and Saikosaponin a. Oncogene 22: 955-963, 2003. 